\title{
Gas entrainment behaviors in the formation and collapse of
}

\section{a ventilated supercavity}

Ashish Karn ${ }^{1,2}$, Roger E.A. Arndt ${ }^{1}$, Jiarong Hong ${ }^{1,3, *}$

1. Saint Anthony Falls Laboratory, $23^{\text {rd }}$ AVE SE, University of Minnesota, Minneapolis, MN, USA 55414.

2. Department of Mechanical Engineering, College of Engineering Roorkee, Uttarakhand, India 247667.

3. Department of Mechanical Engineering, University of Minnesota, Minneapolis, MN, USA 55455.

* Email addresses of the corresponding author: jhong@umn.edu

\begin{abstract}
The present work reports some interesting gas entrainment behaviors in the formation and collapse of a ventilated supercavity under steady and unsteady flow conditions. Our experiments show that the gas entrainment required to establish a supercavity are much greater than the minimum gas entrainment required to sustain it, and these gas entrainment values depend on Froude $(F r)$ number, cavitator size and the flow unsteadiness. Specifically, the measurements of the formation gas entrainment coefficients under different $F r$ numbers indicate that it does not monotonically increase with Fr but displays increasing and decreasing trends in different regimes of Fr. On the other hand, the collapse air entrainment coefficient initially decreases with Fr and then approaches to a constant. Similar trends of formation and collapse gas entrainment coefficient are observed for different cavitator sizes. Moreover, the introduction of unsteady gusts causes a slight monotonic increase in the formation and collapse gas entrainment
\end{abstract}


requirements. Our study points out the crucial factors to be considered in the estimation of gas storage requirements for a ventilated supercavitating vehicle.

Keywords: Supercavitation, ventilation, supercavity formation, supercavity collapse, ventilation hysteresis.

\section{Introduction}

High speed underwater vehicles experience a tremendous amount of flow resistance when moving underwater due to skin friction drag [1-3]. Supercavitation is a special case of cavitation which can be employed to create a bubble of gas/vapor inside water that is large enough to encompass an object (or vehicle) travelling through the water. Supercavitating vehicles are a revolutionary step in the direction of underwater locomotion because it can lead to drag reduction of as high as $90 \%$, facilitating a substantial increase in speed [4]. The phenomenon of supercavitation is generally characterized by non-dimensional parameters such as cavitation number, $\sigma=2\left(P_{\infty}-P_{c}\right) / \rho U^{2}$ and Froude number $\left(F r=U / \sqrt{g d_{c}}\right)$ where $P_{\infty}, \quad P_{c}, \quad \rho$, $U, g$ and $d_{c}$ denote the ambient pressure, the internal cavity pressure, water density, flow velocity, gravitational acceleration and cavitator size, respectively. A supercavity is attained at small $\sigma(\sigma<0.1)$ and this can be accomplished naturally or artificially. A natural supercavity refers to a large attached vapor cavity which is generated by a body travelling at very high speeds (> 90 knots at a depth of $1 \mathrm{~m}$ in open waters) or at low ambient pressure [5]. On the other hand, an artificial or ventilated supercavity is generated by blowing non-condensable gas into the low pressure region near the nose of the vehicle. The non-condensable gas increases the cavity pressure, allowing for low $\sigma$ to be attained at much lower speeds. Ventilated supercavitation has numerous advantages over natural supercavitation viz. greater adaptability 
for vehicle maneuvering and control. Also, some of the typical negative effects encountered in natural cavitation viz. surface damage, buffeting and vibrations etc. are absent in ventilated supercavitation [6]. A vehicle can be optimally ventilated in case of maneuvering etc. to circumvent the possibility of cavity collapse at different stages of vehicle operation.

A typical design strategy of a supercavitating vehicle entails accelerating the vehicle to a high speed at which a natural supercavity can be sustained. The drastic drag reduction required to attain high speeds at the initial launch of the vehicle is achieved by ventilated supercavitation. Further, the operation of a ventilated supercavitating vehicle depends on its ability to supply sufficient gas to fill the cavity at different flow conditions and at different stages of vehicle motion. The ventilation requirements for a ventilated supercavity is characterized by the gas entrainment coefficient at the standard conditions, $C_{\mathrm{Q}}=\dot{Q} / U d_{c}{ }^{2}$, where $\dot{Q}$ denotes the gas ventilation rate at standard conditions (i.e. at a temperature of $273 \mathrm{~K}$ and a pressure of 1 bar. Experimentally $\dot{Q}$ is a direct reading from a mass flow controller). The determination of the gas storage requirements for a ventilated supercavitating vehicle requires information on gas supply rate to form and sustain a steady supercavity at different flow conditions. Thus, it is important to understand the gas entrainment behaviors relating to supercavity formation and collapse.

A number of prior studies have investigated the gas entrainment behavior of ventilated supercavity under a wide range of conditions [5-12].Ventilation hysteresis is intricately related to the formation and collapse gas entrainments and previous studies on ventilation hysteresis have been reported [12]. Ventilation hysteresis refers to a phenomenon whereby the supercavity can be sustained at much lower values of gas entrainment than required to form it, as shown in Fig. 1. 


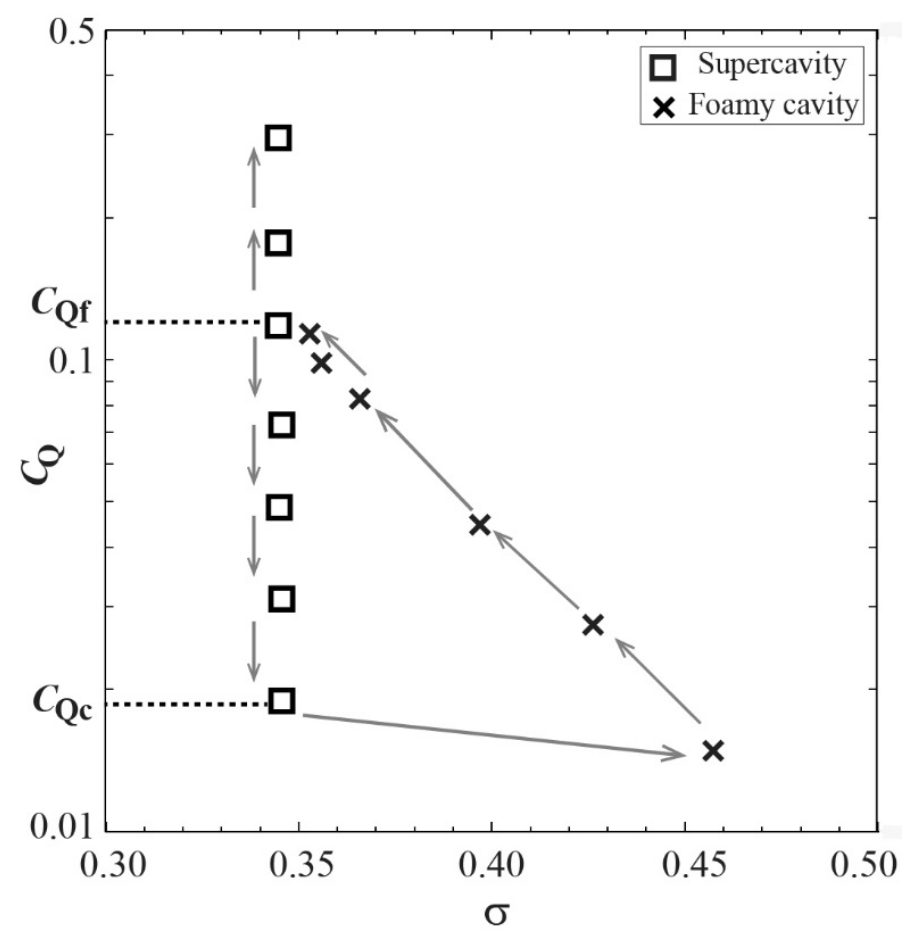

Fig. 1: A typical ventilation hysteresis curve observed in our experiments for a cavitator of $30 \mathrm{~mm}$ in diameter As the above figure shows, a foamy cavity shows a reduction in $\sigma$ (or an increase in length) when $C_{\mathrm{Q}}$ is increased. This process continues until $C_{\mathrm{Q}}$ equals $C_{\mathrm{Qf}}$ (formation gas entrainment coefficient) and a supercavity is established, after which no further reduction in $\sigma$ is possible upon change in $C_{\mathrm{Q}}$. Moreover, the supercavity is maintained even as $C_{\mathrm{Q}}$ drops down to very low values. Eventually, when $C_{\mathrm{Q}}$ drops below $C_{\mathrm{Qc}}$ (collapse gas entrainment coefficient), the supercavity transitions back into a foamy cavity. Recently, Karn et al. [13] discussed the phenomenon of ventilation hysteresis, particularly focusing on the transition of closure modes of a supercavity and relating ventilation hysteresis to the internal flows in a supercavity. However, the gas entrainment rate at the formation and collapse of a supercavity has not yet been examined in detail. 
In this study, we focus our attention on gas entrainment during formation and collapse under steady and unsteady flow conditions, and study the effect of cavitator size on these behaviors. The current paper is structured as follows: $\S 2$ provides the details of experimental methods. In $\S 3$, experimental observations on formation and collapse gas entrainments are reported for steady states along with the effect of cavitator size on these behaviors. Subsequently, we present the behavior of gas entrainments for unsteady states which is followed by a summary and discussion in $\S 4$.

\section{Experimental Setup and Procedures}

Experiments are conducted to measure ventilation flow rates and formation and collapse processes of a ventilated supercavity under different flow conditions. The experiments are carried out in the high-speed water tunnel at the Saint Anthony Falls Laboratory. This water tunnel is a closed recirculating facility with a horizontal test-section of $1.20 \mathrm{~m}$ (Length) $\times 0.19$ $\mathrm{m}$ (Width) $\times 0.19 \mathrm{~m}$ (Height). This tunnel is specifically designed for cavitation and gas ventilation studies and is capable of operating at a maximum velocity of $20 \mathrm{~m} / \mathrm{s}$. Flow unsteadiness can be introduced in the test-section by means of a gust generator as reported in previous studies [14-16]. Fig.2a below shows a schematic of the experimental setup. The gust generator consists of two oscillating slender hydrofoils, which are placed $180 \mathrm{~mm}$ upstream of the cavitator. These hydrofoils oscillate in phase to generate uniform gusts. The motion is activated by a pivot arm that is linked to a flywheel through a connecting arm, which extracts the periodic motion from the motor. The eccentric flywheel allows the generation of gusts of varying amplitudes, represented by angle $\alpha$ as shown in the figure. Time-varying velocity

amplitudes of the periodic gust flows are measured with Laser Doppler Velocimetry as 
described by Lee et al. [16] and it shows that the frequency of the periodic gust flow in the testsection is equal to the oscillation frequency of the gust generator at each flow condition.

A disk cavitator with its back surface facing the incoming flow and mounted downstream of a hydrofoil-shaped strut (referred to as 'backward facing model' in [5], [13] and [14]), is employed in the current experiments. Fig. 2b illustrates the general placement of backwardfacing model within the test section. As it shows, to avoid the interaction between the formed cavity and the strut body, a hydrofoil strut is placed upstream of the cavitator leading to a free closure as reported by Logvinovich [17]. The hydrofoil strut is just thick enough to envelope the ventilation pipe running to the cavitator. The maximum thickness of the hydrofoil, and the external diameter of the ventilation pipe equal $5 \mathrm{~mm}$. High speed videos of the supercavity show that the slender streamlined hydrofoil upstream does not produce significant disturbances on the cavity and only a faint wake signature can be observed. The model has special ventilation ports at the rear of the cavitator to create a supercavity. The gas flow rate is controlled by a mass flow controller and is kept steady during the experiments.

A hypodermic tube used for pressure measurement runs axially inside the ventilation line as shown in Fig. 2c. Pressures in the test section and the supercavity are measured using a Rosemount absolute pressure sensor and a Validyne DP 15-38 differential pressure transducer, respectively. The Validyne pressure transducer is calibrated before each experiment and the pressure lines are purged with compressed gas once before the pressure measurements are recorded at each condition. Uncertainty in the measurements of test-section pressure and supercavity pressure are less than $1 \%$ and $3 \%$, respectively.

During the experiments, the ventilation flow rates for the process of supercavity formation and collapse are measured, and the corresponding high speed videos of this process are recorded 
to obtain physical insights into the phenomena. The measurements of $C_{\mathrm{Qf}}$ and $C_{\mathrm{Qc}}$ are repeated for different cavitator sizes and flow conditions. A cavitator of $30 \mathrm{~mm}$ in diameter is used as the reference (also referred to as the 'reference cavitator' hereafter), and later experiments are repeated with 20 and $40 \mathrm{~mm}$ cavitators to study the effect of change in cavitator size. The tunnel blockages caused by the 20, 30 and $40 \mathrm{~mm}$ cavitators are $9 \%, 14 \%$ and 19\%, respectively. For steady-state experiments, $C_{\mathrm{Qf}}$ and $C_{\mathrm{Qc}}$ are measured at a fixed $F r$, while for the unsteady state experiments, wave-height and gust frequency are fixed as well. The experiments are carried out at gust frequencies $(f)$ of 1,3 and $5 \mathrm{~Hz}$ for each of the gust amplitudes $(\alpha)$ of 2, 4 and 6 degrees.
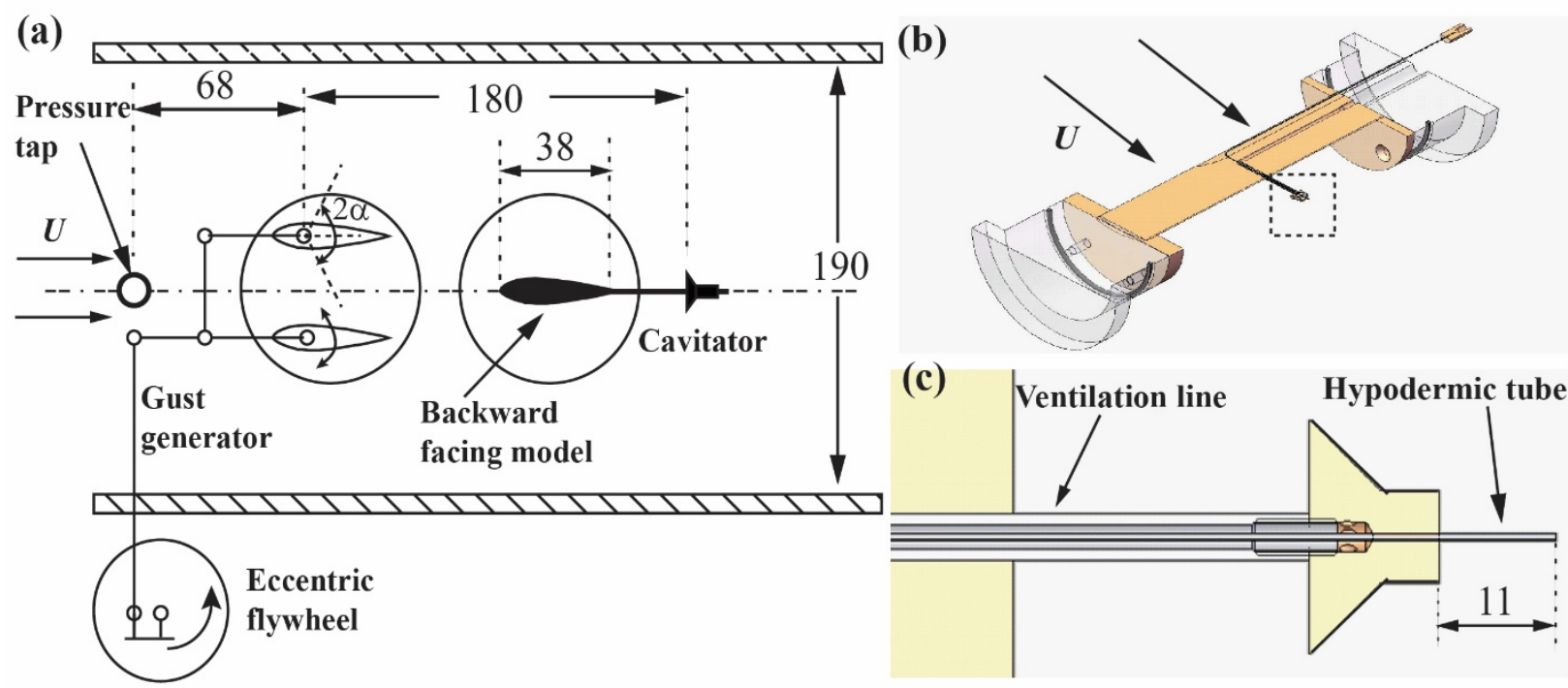

Fig. 2: (a) The experimental setup for studying unsteady ventilated supercavitation. (b) The schematic of the backward-facing cavitator model. (c) The close-up side view of the cavitator. All dimensions are in mm. Adapted from [14]. 


\section{Results and Discussion}

\subsection{Steady state experiments}

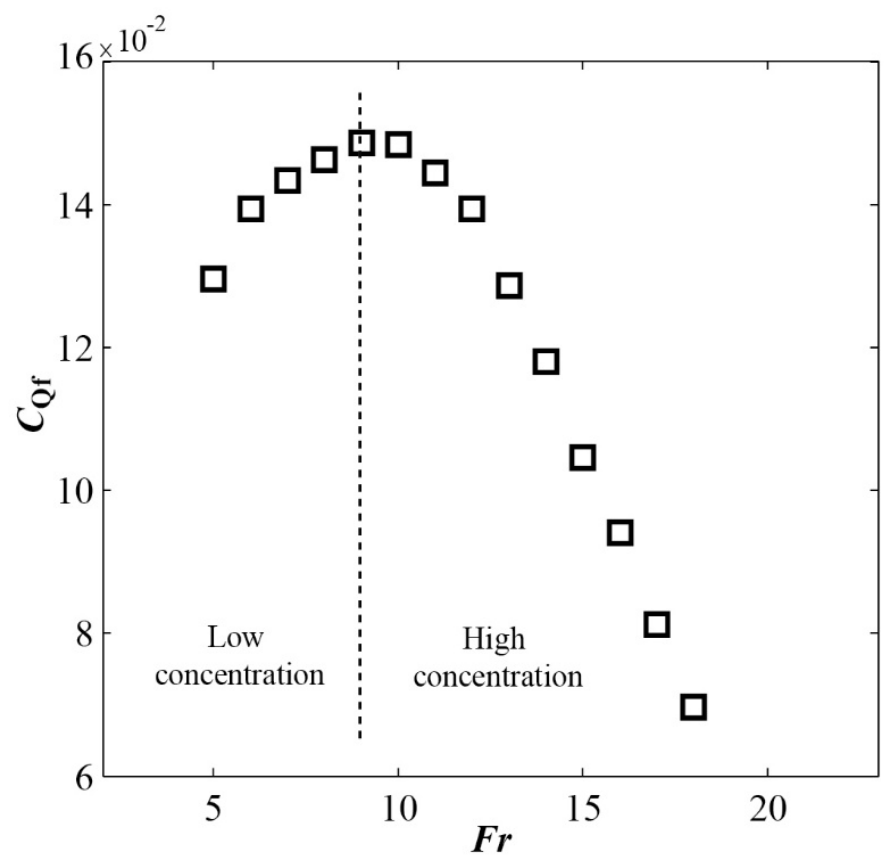

Fig. 3: Dependence of $C_{\mathrm{Qf}}$ upon $F r$ for the reference cavitator

The gas entrainment behaviors at steady state are studied first for the reference cavitator by varying $\mathrm{Fr}$ in the range of $5-25$ (Reynolds number based on the cavitator size, $R e_{d}=$ $\rho U d_{c} / \mu$ ranges from $8.18 \times 10^{4}$ to $4.09 \times 10^{5}$ ). Fig. 3 shows a typical variation of $C_{\mathrm{Qf}}$ with $\mathrm{Fr}$. As the figure shows, with increase in $\mathrm{Fr}, C_{\mathrm{Qf}}$ initially increases, attains a maximum and subsequently decreases. Two separate regimes can be identified around the maximum obtained in this curve, related to the concentration of individual bubbles as will be discussed later. Low $\operatorname{Fr}(\operatorname{Fr}<9)$ corresponds to a low bubble concentration regime, whereas high $\operatorname{Fr}(\mathrm{Fr}>9)$ characterizes a high bubble concentration zone. This trend can be explained based on the assertion that the supercavity formation process is driven by bubble coalescence, as suggested by [13]. In general, for a fixed cavitator size, an increase in Fr implies an increase in flow velocity or turbulence. This increased turbulence generally breaks up the individual bubbles into 
smaller sizes as reported by previous wake studies. Our previous observations have shown that there are lesser number of bubbles at low Fr and an increase in Fr results in smaller individual bubbles, with a consequent increase in the number of bubbles [18-20].With increasing $F r$, the formation of a supercavity in this regime essentially requires the coalescence of these resulting smaller individual bubbles. Thus, there is a greater ventilation requirement to increase the size and number of bubbles to form a coalesced supercavity. Previous studies have reported that an increase in $C_{\mathrm{Q}}$ results in an increment in both number and size of bubbles [18-20]. However, in the high concentration regime, this effect of increased number of bubbles offsets the reduction in the size of individual bubbles. In this regime, the bubble concentration becomes so high that bubbles are closely packed together in the bubble cloud. Due to the restricted free space for bubble movement and the concomitant increase in bubble collision frequency, the coalescence process is favored, resulting in decreased gas entrainment requirement for supercavity formation with increasing $\mathrm{Fr}$.

The dependence of $C_{\mathrm{Qc}}$ upon $F r$ is presented in Fig. 4a. As the figure shows, $C_{\mathrm{Qc}}$ initially decreases sharply with $\mathrm{Fr}$ and then becomes constant. This trend of supercavity collapse can be explained using the postulated framework of supercavity closure presented in [13]. According to [13], the supercavity closure is determined by a balance of pressure inside $\left(P_{\text {in }}\right)$ and outside $\left(P_{\text {out }}\right)$ at the supercavity rear portion. The non-dimensional pressure difference defined as, $\Delta \tilde{P}=$ $2\left(P_{\text {out }}-P_{\text {in }}\right) / \rho U^{2}$, characterizes different supercavity closure modes (e.g. twin-vortex, reentrant jet) as well as the foamy state of a cavity (the supercavity is yet to form). Their data suggested that $\Delta \widetilde{P}$ for a foamy cavity is significantly larger than for a supercavity. In other words, when $P_{\text {out }}$ far exceeds $P_{\text {in }}$, the high pressure difference forces water jet to gush inside the supercavity, causing its collapse. Thus, at a high $\Delta \tilde{P}$, larger gas entrainment is required to inhibit 
the growth of re-entrant jet and maintain a stable supercavity. Further, based on their results, $\Delta \widetilde{P}$ can be estimated from the momentum balance for the supercavity as (neglecting the pressure drop due to viscosity) $\Delta \tilde{P}_{\text {est }}=\sigma-C_{D}\left(d / D_{T}\right)^{2}$, where $D_{T}$ represents the diameter of the water tunnel. As shown in Fig. $4 \mathrm{~b}, \Delta \tilde{P}_{\text {est }}$ exhibits a similar trend with respect to $F r$ as that of $C_{\mathrm{Qc}}$. Thus, larger the $\Delta \tilde{P}_{\mathrm{est}}$, the more the supercavity is susceptible to collapse and consequently a greater gas entrainment is required to prevent such a collapse. It is noteworthy that similar to formation gas entrainment curve, the collapse gas entrainment curve can also be divided into two separate regimes. Our experiments have shown that the regime in which $\Delta \tilde{P}_{\text {est }}$ does not change significantly with $\mathrm{Fr}$ corresponds to supercavities with stable twin-vortex closure at $C_{\mathrm{Qc}}$. On the other hand, the region in which $\Delta \widetilde{P}$ decreases sharply with $F r$ are characterized by other closure modes at $C_{\mathrm{Qc}}$, including re-entrant jet, quad-vortex or unstable closure modes as reported by [13].

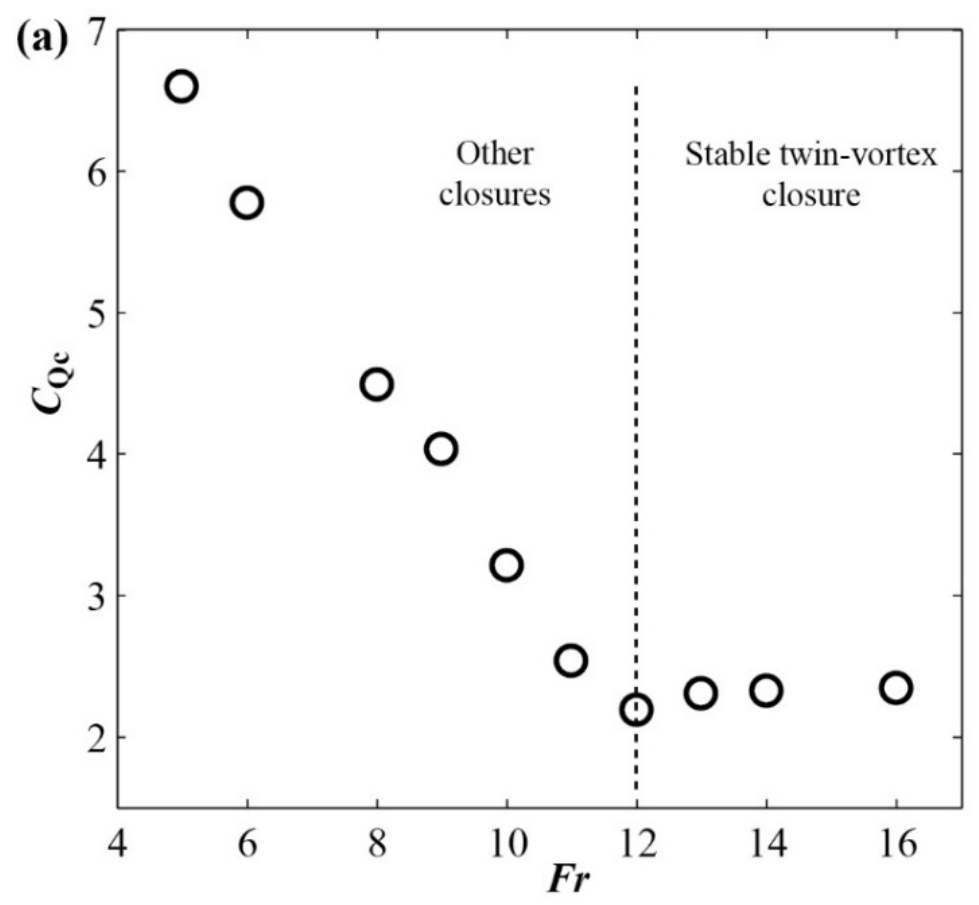




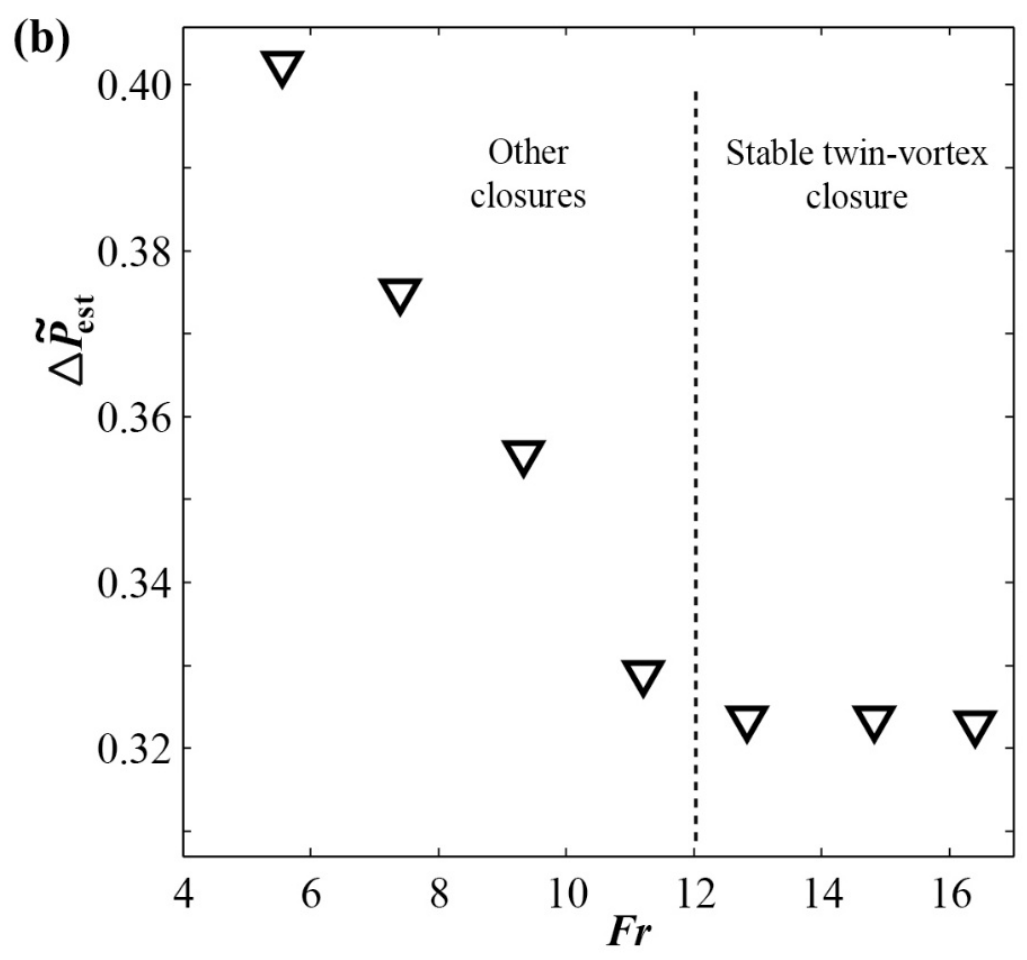

Fig. 4: (a) Dependence of $C_{\mathrm{Qc}}$ upon Fr and (b) Dependence of $\Delta \widetilde{P}_{\text {est }}$ upon $\mathrm{Fr}$ for the reference cavitator. Fr of 4 -16 in the above plots corresponds to an $R e_{d}$ range of $6.54 \times 10^{4}-2.62 \times 10^{5}$.

Next, the effect of cavitator size on $C_{\mathrm{Qf}}$ and $C_{\mathrm{Qc}}$ are examined. Figs. 5 and 6 show the comparison of formation and collapse gas entrainment behavior for three different cavitators, respectively. The figures clearly indicate that a similar trend is observed at a cavitator size of 20 $\mathrm{mm}$ and $40 \mathrm{~mm}$. Further, the curves shift upwards and towards left with an increase in the cavitator size. As pointed out before, for the formation gas entrainment curve, the maximum in this curve represents a transition between low and high bubble concentration regimes. The leftward displacement of the maxima in this curve suggests that the critical $F r$ for such transition decreases with increasing cavitator size. This observation can be ascribed to an increasing wake turbulence (characterized by $R e_{d}$ ) which promotes bubble breakup and consequently results in increased concentration of individual bubbles [20]. The upward movement of the curve can then be attributed to the larger $C_{\mathrm{Qf}}$ required to drive the bubble 
coalescence process into a wider and longer supercavity with increasing cavitator size. On the other hand, for the collapse gas entrainment behavior, the shift in the curves can be attributed to a similar variation of $\Delta \tilde{P}$ with $F r$ as shown in Figs. 4a and 4b. The upward movement of these curves with the increasing cavitator size is related to the greater gas entrainment values required to maintain a larger $\Delta \widetilde{P}$ with increasing cavitator size. This observation is related to the fact that a larger size cavitator results in a supercavity with increase both in cross-sectional area and length, which the gas entrainment coefficient only accounts for the increase in cross- sectional area. As pointed out in Fig. 4b, the flat portion of the $\Delta \widetilde{P}$ vs $F r$ curve corresponds to a stable twin vortex cavity. Thus, the leftward shift in the collapse gas entrainment curve indicates that for a larger cavitator the transition from other supercavity closure modes to a twin vortex cavity mode is established at a low Fr which persists upon further increase in Fr. This indicates that twin vortex is predominantly observed over a larger range of $\mathrm{Fr}$ for a larger cavitator, which has been previously reported and explained by [13].

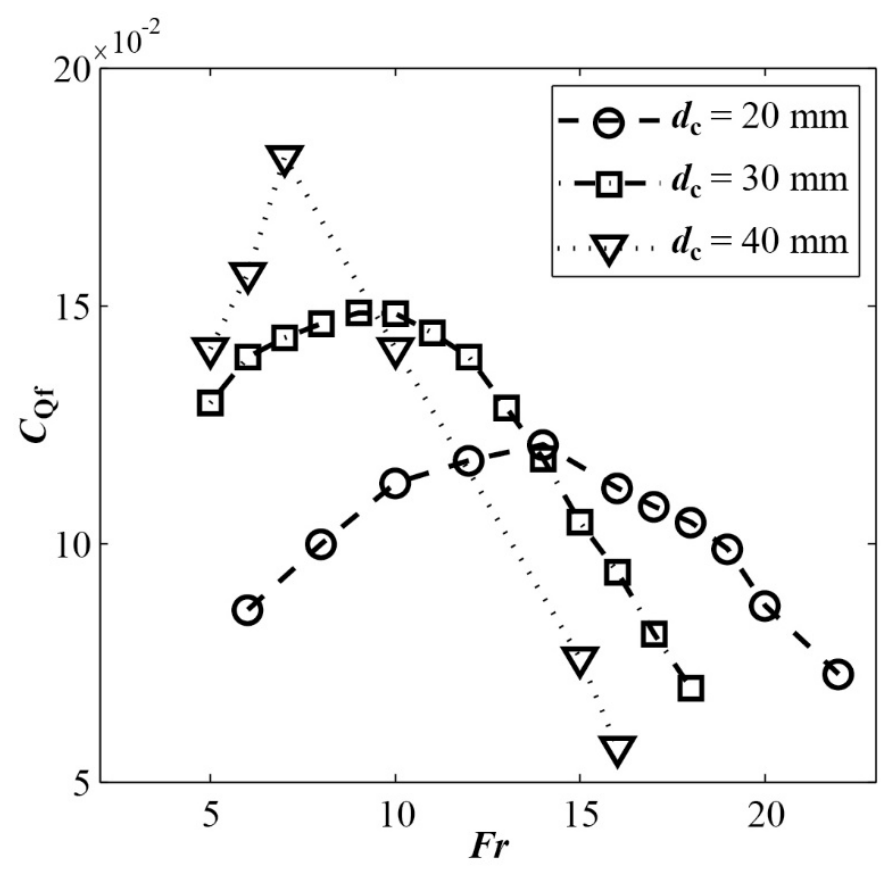

Fig. 5: Comparison of $C_{\mathrm{Qf}}$ vs $F r$ curve for three cavitator sizes 


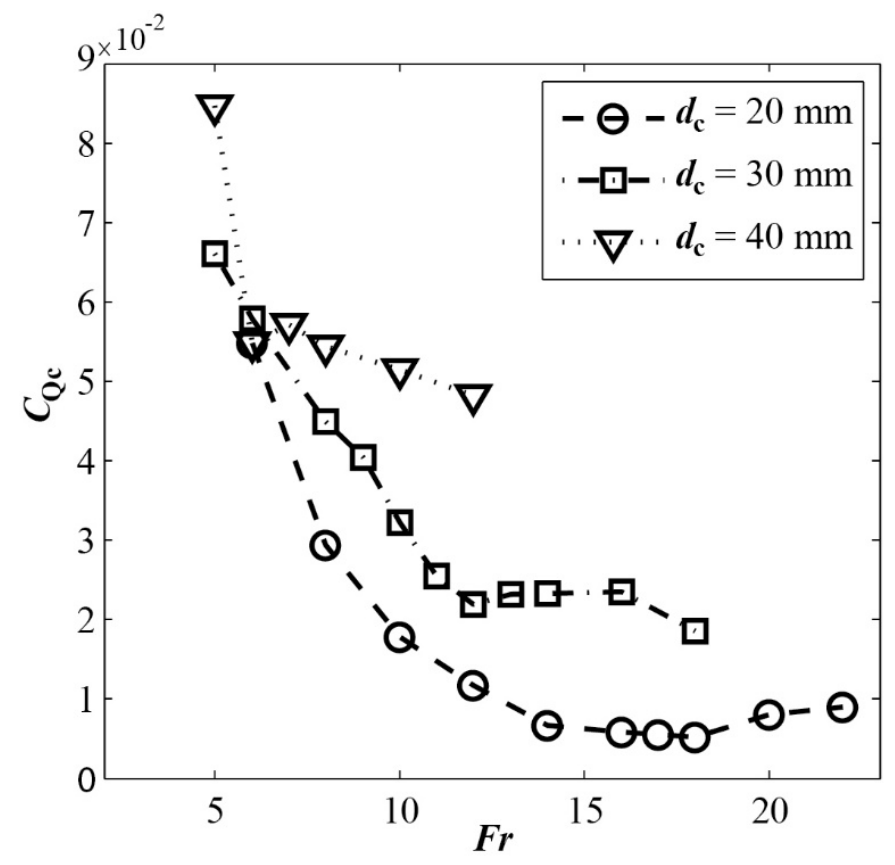

Fig. 6: Comparison of $C_{\mathrm{Qc}} \mathrm{vs} F r$ curve for three cavitator sizes

\subsection{Unsteady state experiments}

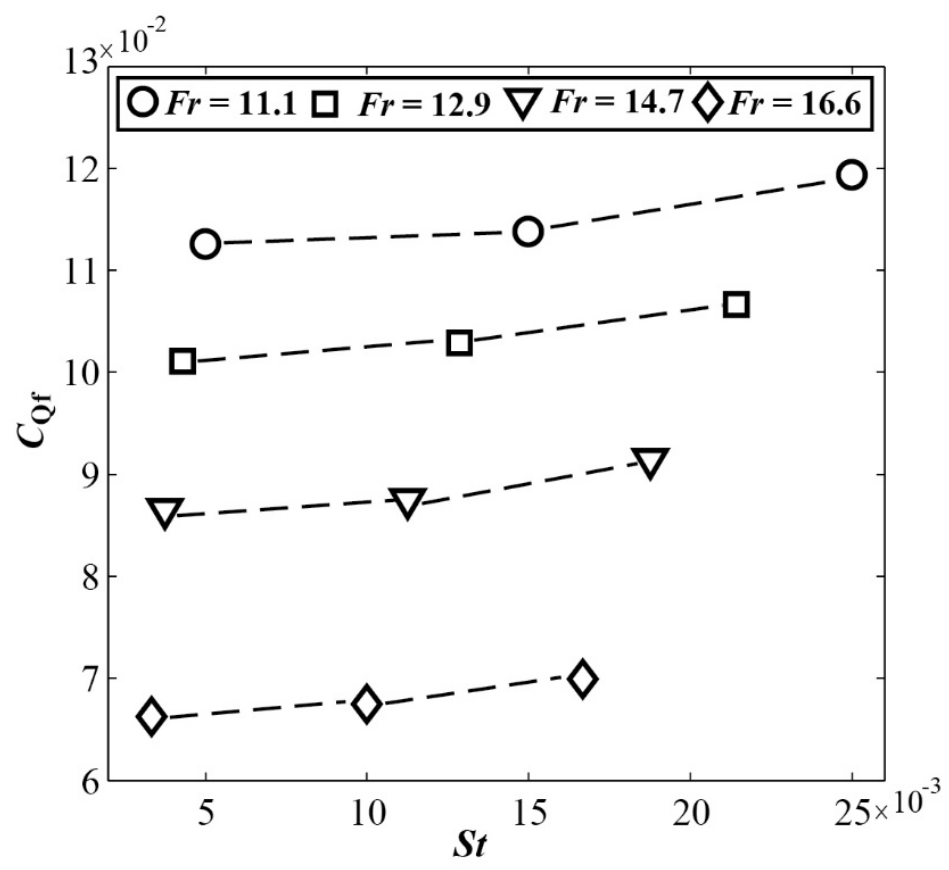

Fig. 7: Variation of $C_{\mathrm{Qf}}$ with $S t$ at different $\mathrm{Fr}$ for the reference cavitator. $R e_{d}$ corresponding to the $F r$ numbers in the plot are $1.82 \times 10^{5}, 2.11 \times 10^{5}, 2.41 \times 10^{5}$ and $2.72 \times 10^{5}$, respectively.

The gas entrainment behaviors under unsteady conditions are studied by first holding Fr and gust amplitude $(\alpha)$ constant and then varying the $S t(=f d / U)$ by changing the gust 
frequencies $(f)$. Fig. 7 shows the formation gas entrainment behavior under different $S t$ values for the reference cavitator at four different $\mathrm{Fr}$ numbers. As Fig. 7 shows, $C_{\mathrm{Qf}}$ increases monotonically with increase in $S t$ at a fixed $F r$. This trend in $C_{\mathrm{Qf}}$ with $S t$ indicates that the introduction of unsteady gusts in the flow impacts the bubble coalescence process by imposing a lateral velocity on the bubbles. This imposed lateral motion on bubbles makes it more difficult for the coalescence of individual bubbles into a supercavity, thus increasing the $C_{\mathrm{Qf}}$ requirement with an increase in St. It is noteworthy that unlike coalescence events in the flow, breakup events is much less affected by the introduction of unsteadiness since the timescales associated with bubbles breakup are substantially smaller than those of the unsteady gusts.

Fig. 8 shows the effect of the variation of gust amplitudes upon $C_{\mathrm{Qf}}$ at a fixed $F r$ and $S t$. As the figure shows, $C_{\mathrm{Qf}}$ increases monotonically with increase in $\alpha$. High speed videos of the supercavity indicates that an increase in gust amplitude results in the bubbles being spread across a larger lateral cross-section (reducing the local void fraction), which decreases the collision frequency of bubbles and consequently reduces coalescence efficiency. Thus, a greater gas entrainment is required to drive the coalescence of individual bubbles into a supercavity. 


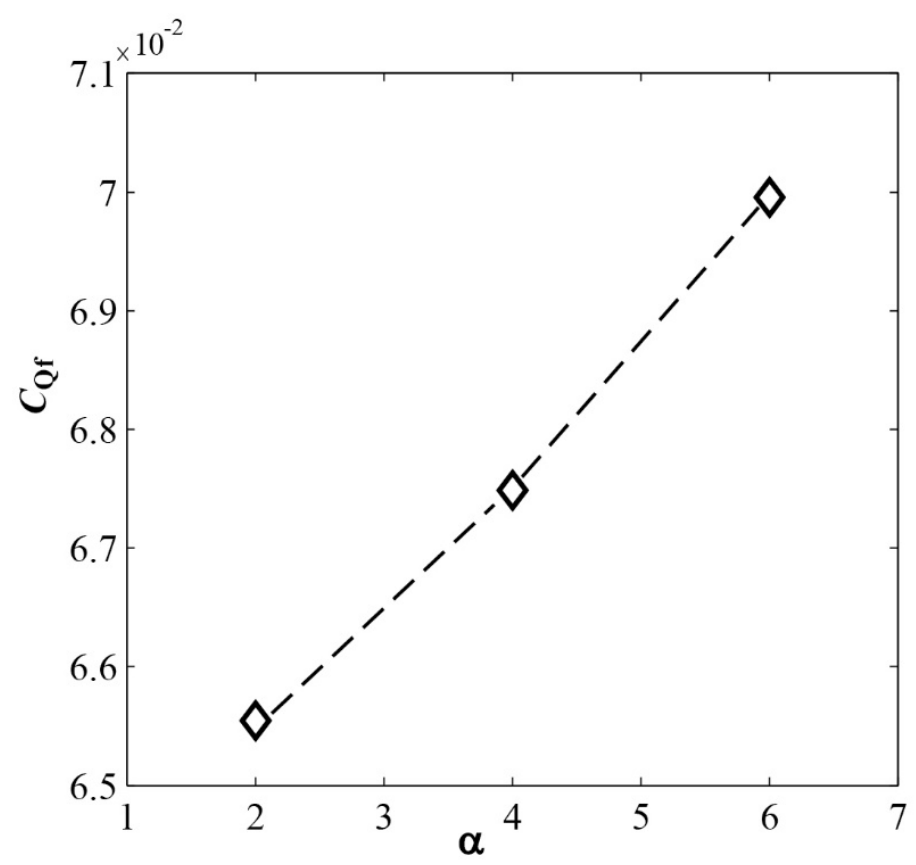

Fig. 8: Variation of $C_{\mathrm{Qf}}$ with $\alpha$ at different $\mathrm{Fr}$ for the reference cavitator

Next, experiments are conducted to study the behavior of collapse gas entrainment under unsteady states for the reference cavitator. Fig. 9 shows the variation of $C_{\mathrm{Qc}}$ with $S t$ at three different $F r$ numbers. As the figure shows, at a fixed $F r, C_{\mathrm{Qc}}$ increases monotonically with increase in St. This monotonic increase in $C_{\mathrm{Qc}}$ with $S t$ can be explained as follows. As pointed out before, the process of supercavity collapse is preceded by a transition to a re-entrant jet closure, which makes the supercavity very unstable, particularly in an unsteady flow. A previous study [17] has shown that the introduction of unsteady gusts in the flow lead to pressure fluctuations at the closure, intermittently causing a re-entrant jet closure. As St increases, a larger $C_{\mathrm{Qc}}$ is required to counteract the rapid pressure fluctuations occurring at reentrant jet closure and sustain the supercavity. A similar explanation can also be given for a larger $C_{\mathrm{Qc}}$ obtained at a higher $F r$, but nearly same St. Previous studies (For instance, [21]) have shown that, the re-entrant jet velocity is approximately equal to the incoming liquid velocity. Thus, an increase in $\mathrm{Fr}$ for the same cavitator size implies an increase in re-entrant jet velocity 
and thus a larger $C_{\mathrm{Qc}}$ is needed to inhibit the re-entrant jet from reaching the cavity upstream and causing cavity collapse.

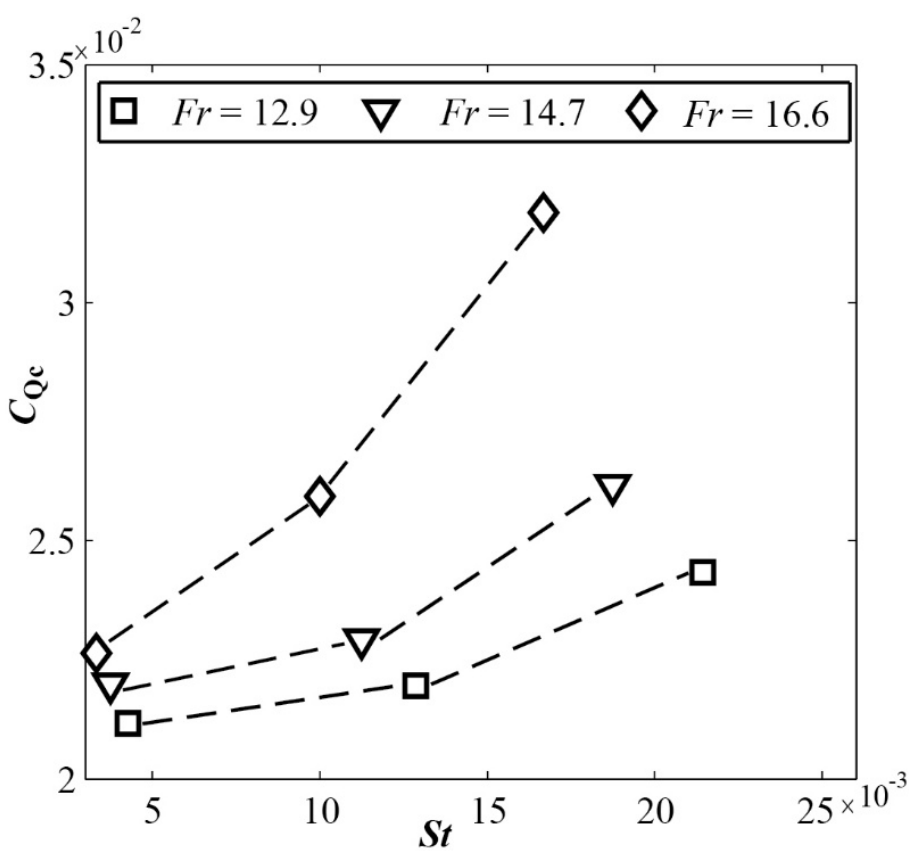

Fig. 9: Variation of $C_{\mathrm{Qc}}$ with $S t$ at different $\mathrm{Fr}$ numbers for the reference cavitator. $R e_{d}$ corresponding to the $\mathrm{Fr}$ numbers in the plot are $2.11 \times 10^{5}, 2.41 \times 10^{5}$ and $2.72 \times 10^{5}$, respectively.

The effect of the variation of gust amplitudes upon $C_{\mathrm{Qc}}$ is found to be similar to $C_{\mathrm{Qf}}$. Fig.10 shows the effect of the variation of gust amplitudes upon $C_{\mathrm{Qc}}$ at a fixed Fr and St. From Fig. 10, it can be seen that $C_{\mathrm{Qc}}$ increases monotonically with increase in $\alpha$. Again, this observation can be related to the increase in the extent of pressure fluctuations at the supercavity rear with the increase in $\alpha$. Thus, a larger $C_{\mathrm{Qc}}$ is required to counteract the rapid pressure fluctuations occurring at the supercavity rear portion and sustain the supercavity. 


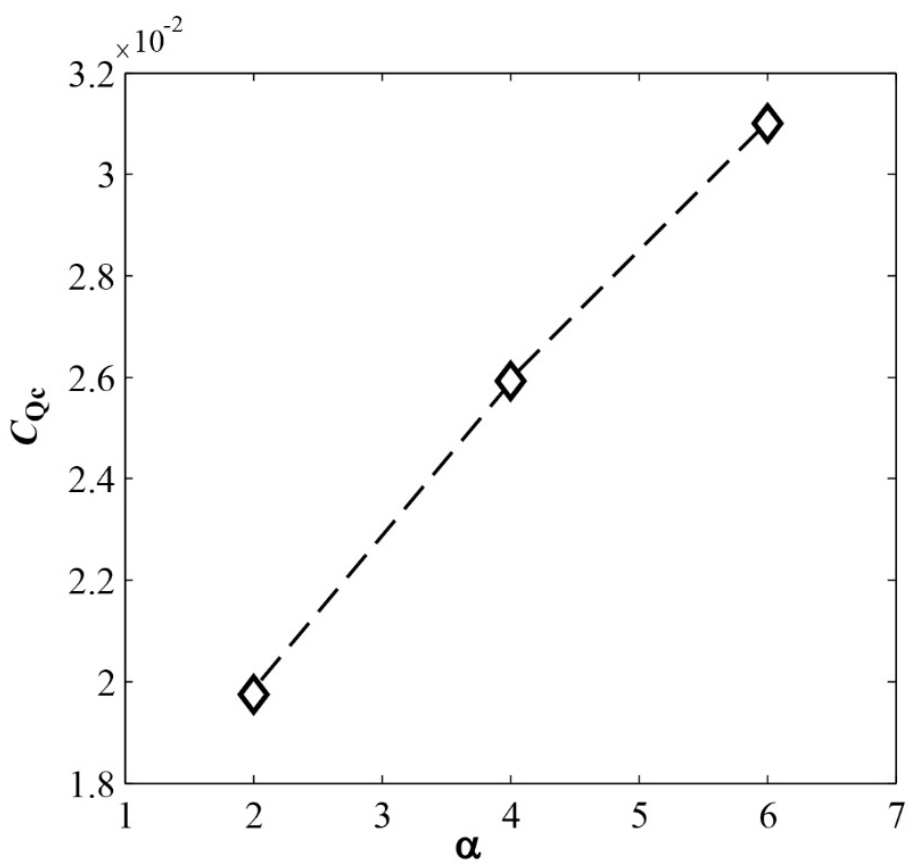

Fig. 10: Variation of $C_{\mathrm{Qc}}$ with $\alpha$ at different $F r$ for the reference cavitator

\section{Summary and Conclusions}

In the current study, some interesting insights into the gas entrainment behaviors in the formation and collapse of a ventilated supercavity are presented for steady and unsteady flow conditions. Our experiments have shown that the gas entrainment required to establish a supercavity is much greater than the minimum gas entrainment required to sustain it. Further, these gas entrainment values depend on $F r$, cavitator size and the flow unsteadiness. Specifically, the measurements of the formation gas entrainment coefficients under different $\mathrm{Fr}$ numbers indicate that it does not monotonically increase with $\mathrm{Fr}$ but displays increasing and decreasing trends in different regimes of $F r$. These trends are possibly caused by the variation in coalescence efficiency with bubble concentration at different flow conditions. On the other hand, the collapse air entrainment coefficient initially decreases with $F r$ and then approaches to a constant. This trend has been shown to be related to the change in the non-dimensional 
pressure inside and outside the cavity at the supercavity rear portion. Similar trends of formation and collapse gas entrainment coefficient have been observed for different cavitator sizes.

Our unsteady state experiments have shown that both the introduction of unsteadiness and the increase in gust amplitudes cause a slight monotonic increase in the formation and collapse gas entrainment requirements. For the supercavity formation, the observed trends are related to lateral motion and expansion of the bubbles induced by the unsteadiness, which reduces the coalescent efficiency of bubbles. For the collapse case, the trends are determined by the pressure fluctuations occurring at the supercavity closure. These findings have far-reaching implications in the operation of underwater supercavitating vehicles. Specifically, knowledge of the occurrence of ventilation hysteresis and its dependence upon flow conditions can drastically reduce the ventilation demand for extended underwater missions. Our study points out the crucial factors to be considered in the estimation of gas storage requirements for such a ventilated supercavitating vehicle.

\section{Nomenclature}

\begin{tabular}{|c|l|c|l|l|l|}
\hline$P_{\infty}$ & Test-section pressure & $\dot{Q}$ & Gas ventilation rate & $C_{\mathrm{Qc}}$ & Collapse coefficient \\
\hline$P_{\mathrm{C}}$ & Internal cavity pressure & $\sigma$ & Cavitation number & $f$ & Gust frequency (Hz) \\
\hline$\rho$ & Density of water & $F r$ & Froude number & $\alpha$ & Gust amplitude (deg) \\
\hline$U$ & Incoming liquid velocity & $C_{\mathrm{Q}}$ & Gas-entrainment & $S t$ & Strouhal number \\
\hline$d$ & Cavitator diameter & $C_{\mathrm{Qf}}$ & Formation & $D_{T}$ & Water tunnel diameter \\
\hline
\end{tabular}

\section{Acknowledgments}

The authors acknowledge support by the Office of Naval Research (Program manager, Dr. Ronald Joslin) under Grant \# N000140910141. We acknowledge the help of undergraduate student Mr. Roger Borgeson for his help in carrying out the experiments. 


\section{References}

[1] J.P. Franc and J.M. Michel, Fundamentals of Cavitation, Kluwer Academic Publishers, Dordrecht, 2004.

[2] C.E. Brennen, Cavitation and Bubble Dynamics, Oxford University Press, Oxford, 1995.

[3] Vlasenko, Yu. D., Savchenko, G. Yu., "Study of the parameters of a ventilated supercavity closed on a cylindrical body" from "Supercavitation: Advances and Perspectives A collection dedicated to the 70th jubilee of Yu. N. Savchenko," Springer Science \& Business Media, Jan. 2012, pp. $201-214$.

[4] I.N. Kirschner, N.E. Fine, J.S. Uhlman and D.C. Kring, "Numerical modeling of supercavitating flows”, Technical report, DTIC, (2001).

[5] E. Kawakami and R. E. A. Arndt, "Investigation of the behavior of ventilated supercavities,” J. Fluids Eng. 133 (9) (2011) 091305-1 - 091305-11.

[6] M. P. Kinzel, J. W. Lindau and R. F. Kunz, "Air entrainment mechanisms from artificial supercavities: Insight based on numerical simulations," Proceedings of the $7^{\text {th }}$ International Symposium on Cavitation, CAV 2009 (2009), 136, Ann Arbor, Michigan, USA.

[7] Epshtein, L. A., "Characteristics of ventilated cavities and some scale effects," Unsteady water flow with high velocities, Proceedings of IUTAM Symposium, Leningrad, 1973, 173-185.

[8]Y.Savchenko, "Supercavitation-problems and perspectives," http://resolver.caltech.edu/cav2001: lecture. 003 (2001).

[9] J. H. Spurk, “On the gas loss from ventilated supercavities," ActaMechanica 155 (3-4), 2002, 125-135.

[10] R. Cox and W. Clayden, “Air entrainment at the rear of a steady cavity,” Proceedings of the symposium on cavitation in hydrodynamics, London, 1955.

[11] I.J. Campbell, and D. V. Hilborne, "Air entrainment behind artificially inflated cavities," Proceedings of the Second Symposium on Naval Hydrodynamics, Washington,1958.

[12] E. Kawakami, "Investigation of the behavior of ventilated supercavities," MS Thesis (2010)University of Minnesota Twin cities.

[13] A. Karn, R.E.A. Arndt, and J. Hong, "An experimental investigation into supercavity closure mechanisms,” J. Fluid Mech. 789 (2016) 259-284.

[14] A. Karn, R.E.A. Arndt, and J. Hong, "Dependence of supercavity closure upon flow unsteadiness,” Exp. Therm. Fluid Sci. 68 (2015) 493-498.

[15] J. Kopriva, R.E.A Arndt, and E. L. Amromin, "Improvement of hydrofoil performance by partial ventilated cavitation in steady flow and periodic gusts," J. Fluids Eng. 130 (3) (2008) 031301-1 - 031301-7. 
[16] S. J. Lee, E. Kawakami, and R.E.A. Arndt, "Investigation of the Behavior of Ventilated Supercavities in a Periodic Gust Flow,” J. Fluids Eng. 135 (8) (2013) 081301-1 - 081301-13.

[17] G. V. Logvinovich, “Hydrodynamics of free-boundary flows,” Halsted Press, 1973.

[18] A. Karn, C. Ellis, J. Hong, and R.E.A. Arndt, "Investigation into the turbulent bubbly wake of a vented hydrofoil: moving towards improved turbine aeration techniques," Exp. Therm. Fluid Sci. 64 (2015) 186-195.

[19] A. Karn, C. Ellis, C. Milliren, J. Hong, D. Scott, R.E.A. Arndt, and J. Gulliver, "Bubble size characteristics in ventilated hydrofoils with two aeration configurations," Int. J. Fluid Machinery Syst. 8 (2) (2015) 73-83.

[20] A. Karn, S. Shao, R.E.A. Arndt, and J. Hong, "Bubble coalescence and breakup in turbulent bubbly wake of a ventilated hydrofoil,” Exp. Therm. Fluid Sci. 70 (2016) 397-407.

[21] W. Zou, K. P. Yu, R. E. A. Arndt, G. Zhang, and Z. W. Li, "On the shedding of the ventilated supercavity with velocity disturbance,” Ocean Eng.57 (2013) 223-229. 